American Journal of Applied Sciences 6 (8): 1502-1508, 2009

ISSN 1546-9239

(C) 2009 Science Publications

\title{
An Efficient Low Complexity Lossless Coding Algorithm for Medical Images
}

\author{
S.E. Ghrare, M.A. Mohd. Ali, K. Jumari and M. Ismail \\ Department of Electrical and Electronic Engineering, Faculty of Engineering and Built Environment, \\ University Kebangsaan Malaysia 43600 UKM, Bangi, Malaysia
}

\begin{abstract}
Problem statement: Nowadays a large number of various medical images are generated from hospitals and medical centers with sophisticated image acquisition devices, the movement toward digital images in radiology presents the problem of how to conveniently and economically store, retrieve and transmit the volume of digital images. Thus digital image data compression is necessary in order to solve this problem. So in a wide range of medical applications such as disease diagnostic and during the compression process, the loss of information is unacceptable; hence medical images are required to be at high resolution as possible. Instead of lossy compression with relatively high compression ratio, mathematical lossless compression methods are favored in this field. Approach: In this study, an efficient new lossless image coding algorithm using a simple technique was presented. Our coding algorithm was based on pixel redundancy reduction by formulating two matrices only, which were Gray Scale Matrix (GSM) and Binary Matrix (BM). These matrices had been used for coding and decoding processes. Results: Results showed that the maximum compression ratio achieved using the proposed method was $4: 1$, which was more efficient than the present lossless techniques, moreover the computational complexity is greatly simplified; therefore producing very fast coding and decoding. Conclusion: This algorithm was most suitable for those images where lossy compression was avoided such as medical images used for teleradiology and other telemedicine purposed and it can be applied to other medical modalities.
\end{abstract}

Key words: Lossless compression, medical image, teleradiology, telemedicine

\section{INTRODUCTION}

Medical imaging is a powerful and useful tool for radiologists and consultants, allowing them to improve and facilitate their diagnosis. Worldwide, $\mathrm{X}$-ray images represent $60 \%$ of the total amount of radiological images, the remaining consists of more newly developed image modalities such as Computed Tomography (CT), Magnetic Resonance Imaging (MRI), Ultrasound (US), Positron Emission Tomography (PET), Nuclear Medicine (NM) and Digital Subtraction Angiography (DSA) $)^{[1,2]}$.

Image communication systems for medical images have bandwidth and image size constraints that result in time-consuming transmission of uncompressed raw image data. Thus image compression is a key factor to improve transmission speed and storage, but it risks losing relevant medical information ${ }^{[3]}$. It exploits common characteristics of most images that are the neighboring picture elements or pixels are highly correlated $^{[4]}$. It means a typical still image contains a large amount of spatial redundancy in plain areas where adjacent pixels have almost the same values. Compression techniques can be categorized into lossy and lossless. Lossy techniques permit some signal degradation and provide higher compression ratios in comparison with lossless techniques. Lossless compression techniques do not permit any loss of information and allow the original signal to be recovered exactly.

The choice of the compression method, lossy or lossless, depends on the application. For example, in applications dealing with speech signals and video television images, where some loss of information can be tolerated, lossy compression methods can be used. On the other hand in a wide range of medical applications and under special circumstances such as disease diagnostic, the loss of information is unacceptable; hence medical images are required to be at high resolution as possible. Thus, rather than lossy compression with relatively high compression ratio, mathematical lossless compression methods are favored in this field ${ }^{[5]}$.

Corresponding Author: S.E. Ghrare, Department of Electrical and Electronic Engineering, Faculty of Engineering and Built Environment, University Kebangsaan Malaysia 43600 UKM, Bangi, Malaysia 
In this study we present a new coding algorithm for medical images. This algorithm is absolutely lossless and based on pixel redundancy reduction using only two matrices for coding and decoding processes without affecting the quality of the resultant reconstructed image.

Background: Compression is the process of storing or packing data in a format that requires less space than the initial file. Compression takes an input $\mathrm{X}$ and generates a representation $X_{C}$ that hopefully requires fewer bits. There is a reconstruction algorithm that operates on the compressed representation $\mathrm{X}_{\mathrm{C}}$ to generate the reconstruction $\mathrm{Y}$ and the performance of the algorithm depends on the type of the compression technique.

Table 1 shows the need for sufficient storage space, large transmission bandwidth and long transmission time for image, audio and video data. At the present state of technology, the only solution is to compress multimedia data before its storage and transmission and decompress it at the receiver for play back.

Teleradiology without compression: In teleradiology applications, medical image compression is essential despite of rapid growth in digital communication systems performance, mass storage and processors speed. The requirement for data storage capacity and bandwidth continue to exceed the capability of available technologies.

A radiologist would need to review personally all the medical images at a computer workstation to make sure that all possible information that might be clinically important is reviewed at all combinations of window level settings. Table 2 that data files of images would become much larger and consequently retrieval of images from archives will be slow. So, without mathematical image compression, digital imaging would be more expensive, less practical and less attractive for the advantages it offers. Either transmission time would be much longer or telecommunication equipment and line charges would be more expensive ${ }^{[8]}$. Therefore, teleradiology and telemedicine would in many situations be impractical, unacceptably slow and expensive ${ }^{[9]}$.

Review of related work: In radiology the discussion of image compression is divided into three separate categories: Compression before primary diagnosis for rapid transmission, compression after primary diagnosis for long-term archiving and compression for database browsing where progressive would be useful.

In the last two decades several scientific studies have been performed to determine the degree of compression that maintains the physical and diagnostic image quality. Hundreds of research studies have been published during that period of time reviewing the existing techniques or presenting the new advances in the field of medical image compression in both categories of lossy and lossless. Short review to some published study and research studies since 1990 is presented.

In 1991, a group from Japan carried out an analysis study of computed radiography images and they concluded that plain Computed Radiography (CR) chest images with a compression ratio 10:1 are acceptable using discrete cosine transform technique ${ }^{[10]}$.

In 1994, Gillespy and Rowberg studied the compression effect using the cosine transform on hand radiographs. They concluded that no statistically significant loss of diagnostic quality was detected for 8 or 7-bit compress images with average compression ratios of $16: 1$ and $28: 1^{[11]}$.

In 1995, Breeuwer et $a .^{[12]}$ discussed the diagnostic consequences of compression using cosine transform. They reported that acceptable compression ratio for image size of 2048, 1024 and 512 pixels were $25: 1,20: 1$ and 10:1 respectively based on a mean square error of $0.02 \%$. The researchers concluded that reconstructed images from compressed image data with a compression ratio of 4:1-16:1 did not result in excessive visual degradation and therefore this technique was suitable for compression of diagnostic images $^{[12]}$.

Table 1: Memory space, transmission bandwidth and transmission time requirements for uncompressed multimedia data files ${ }^{[6]}$

\begin{tabular}{|c|c|c|c|c|c|}
\hline Multimedia data & Size & $\begin{array}{l}\text { Resolution } \\
\text { bit/pixel }\end{array}$ & $\begin{array}{l}\text { Uncompressed } \\
\text { size (bytes) }\end{array}$ & $\begin{array}{l}\text { Transmission } \\
\text { bandwidth }\end{array}$ & $\begin{array}{l}\text { Transmission } \\
\text { time }\end{array}$ \\
\hline A paper of text & $11 " \times 8.5 "$ & Varying resolution & $4-8 \mathrm{~KB}$ & $32-64 \mathrm{~Kb}_{\text {page }}{ }^{-1}$ & $1.1-2.2 \mathrm{sec}$ \\
\hline Telephone quality speech & $10 \mathrm{sec}$ & 8 & $80 \mathrm{~KB}$ & $64 \mathrm{~Kb} \mathrm{sec}^{-1}$ & $22.2 \mathrm{sec}$ \\
\hline Gray scale image & $512 \times 512$ & 8 & $262 \mathrm{~KB}$ & $2.1 \mathrm{Mb}$ image $^{-1}$ & $1 \mathrm{~min} 13 \mathrm{sec}$ \\
\hline Color image & $512 \times 512$ & 24 & $786 \mathrm{~KB}$ & $6.29 \mathrm{Mb}$ image $^{-1}$ & $3 \mathrm{~min} 39 \mathrm{sec}$ \\
\hline Medical image & $2048 \times 1680$ & 12 & $5.16 \mathrm{MB}$ & $41.3 \mathrm{Mb} \mathrm{image}^{-1}$ & $23 \mathrm{~min} 54 \mathrm{sec}$ \\
\hline SHD image & $2048 \times 2048$ & 24 & $12.58 \mathrm{MB}$ & $100 \mathrm{Mb}$ image $^{-1}$ & $58 \mathrm{~min} 15 \mathrm{sec}$ \\
\hline Full-motion video & $\begin{array}{l}640 \times 480 \\
\left(30 \text { frames sec }^{-1}\right)\end{array}$ & 24 & $1.66 \mathrm{~GB}$ & 221 Mbimage $^{-1}$ & 5 days $8 \mathrm{~h}$ \\
\hline
\end{tabular}


Am. J. Applied Sci., 6 (8): 1502-1508, 2009

Table 2: Sizes and storage requirement for radiological images ${ }^{[7]}$

\begin{tabular}{lllll}
\hline Modality & $\begin{array}{l}\text { Image size } \\
\text { (pixels) }\end{array}$ & $\begin{array}{l}\text { Resolution } \\
\text { (bits/pixel) }\end{array}$ & $\begin{array}{l}\text { Average } \\
\text { images } \\
\text { per exam }\end{array}$ & $\begin{array}{l}\text { Average storage } \\
\text { requirement } \\
\text { per exam (MB) }\end{array}$ \\
\hline CT & $512 \times 512$ & 12 & 30 & 15.0 \\
MRI & $256 \times 256$ & 12 & 50 & 6.5 \\
DSA & $1000 \times 1000$ & 8 & 20 & 20.0 \\
US & $512 \times 512$ & 6 & 36 & 9.0 \\
NM & $128 \times 128$ & 8 & 26 & 0.4 \\
Computed & $2000 \times 2000$ & 10 & 4 & 32.0 \\
radiography & & & & \\
Digitized film & $4000 \times 4000$ & 12 & 4 & 128.0 \\
\hline
\end{tabular}

In 1997, Erickson et al. ${ }^{[13]}$ found that a lossy compression of 40:1 or more could be used without perceptible loss in the representation of anatomical structures.

In 1999, Zhang and $\mathrm{Wu}^{[14]}$ proposed a contentbased compression of mammograms with a modified wavelet based JPEG2000 using biorthogonal wavelets. The algorithm retained $90 \%$ of marked micro calcifications and compression rates of up to 12:1 (for $15 \%$ ROI segmentation) and 18.1 (for $10 \%$ ROI segmentation) were noted.

In 2000, a comparison study between the new JPEG2000 and the conventional JPEG lossy compression was presented with application to a large series of CT and MRI images of the head. The author found that JPEG and JPEG2000 were not substantially different in the maximum acceptable compression ratio ${ }^{[15]}$.

In 2001, a comparative study of image compression between JPEG and wavelet was made. The researchers of this study concluded that the wavelet could achieve 2-3 times higher compression efficiency than JPEG for higher compression ratios without compromising image quality. The weakness of this study is that it performed on only one medical image modality, the X-ray. Moreover, the quality of the reconstructed images has been evaluated using the objective measures without the subjective measure which is still a method commonly used in measuring image quality ${ }^{[16]}$.

In 2002, the same researchers published another study involving both objective and subjective measures to evaluate compressed medical images. They concluded that a compression ratio 20:1-30:1 is acceptable for computed tomography images. This study was also applied for one type image modality only ${ }^{[17]}$. In the same year, Hui and Besar studied the compression performance of the new JPEG-2000 and the more conventional JPEG applied on medical images. The parameters used in their study for compression include the compression efficiency, Peak Signal-to-Noise Ratio (PSNR), Picture Quality Scale (PQS) and Mean Opinion Score (MOS). Their study shows that JPEG-2000 compression is more acceptable and superior compared to JPEG for lossy compression $^{[18]}$.

Also in the 2002, Carlender and Christopoulos presented a Region Of Interest (ROI) coding method for image compression using wavelets. This method was applied for ROI areas varying from $8-25 \%$ and they noted a compression ratio of $16: 1$ up to $50: 1$ on different non medical images such as Lena, Woman ${ }^{[19]}$.

A $2003 \mathrm{r}$ research suggested that the upper limit for mammograms compression to be in the range 35-40:1. These results were based on the Receiver Operating Characteristics (ROC), as well as PSNR, RMSE and CR of 68 mammogram images ${ }^{[20]}$.

In 2004, a wavelet transform based JPEG2000 was used for testing 40 mammogram images at different compression ratios 1:1, 10:1, 20:1 and 30:1, with results reviewed by seven observers. No significant compression loss was noted up to 20:1 compression ratio. The compression was applied to the images as a whole and no prior segmentation was performed ${ }^{[21]}$.

In 2005, an efficient medical image compression scheme was proposed, consisting of two stages. In the first stage, a Differential Pulse Code Modulation (DPCM) is used to decorrelate the raw image data and in the second the Huffman coding method is used to encode the residual image. The compression ratio achieved was higher than the existing lossless JPEG, ranging from 1.64 up to $3.12^{[22]}$.

In 2006, a new lossless compression technique was proposed for medical images. It was a hybrid of lossless and lossy techniques using neural network vector quantization and Huffman coding. This technique was applied on CT images with a performance of 5 10 compression ratio ${ }^{[23]}$.

Also in 2006, another lossless medical image compression technique based on redundancy analysis was proposed. This technique was based on the segmentation of the image into Variable Block Size (VBS) and then the smoothness and the similarity of the blocks were determined. This method works better by $10-40 \%$ than other compression methods such as Huffman, JPEG-LL and Lossless JPEG-2000 $0^{[24]}$

In 2007, another new compression method was proposed based on using wavelet transform, Differential Pulse Code Modulation (DPCM) and adaptive run length coding. The scheme utilizes biothogonal wavelet transforms to decompose the image signal, then uses run-length coding to compress the detail sub-bands ${ }^{[25]}$. The weakness of this method comes from its computational load and complexity and the results obtained from applying this method on nonmedical images have not been compared with the existing methods. 
In 2008, Yao et al. ${ }^{[26]}$ Introduced a new compression method based on row by row pixel classification and LZW encoding technique. In this method, pixels of an image are classified row by row; pixels similar in value are gathered and encoded by LZW. The weakness of this method is its decoding process complexity. Moreover, it has been tested only on non medical images.

\section{MATERIALS AND METHODS}

Our algorithm is based on only two matrices, binary matrix and grayscale matrix. The main steps of the proposed algorithm are as follows:

Step 1: Read the original image matrix [OR]

Step 2: Construct the Binary Matrix [BM] and Grayscale Matrix [GSM] based on the following steps

Step 3: Compare each pixel in the matrix [OR] with the previous pixel in the same matrix as shown in Fig. 1

Step 4: The binary matrix elements are calculated as follows:

$$
[\mathrm{BM}]_{\mathrm{i}, \mathrm{j}}=\left[\begin{array}{ll}
0 & \text { if } \quad[\mathrm{OR}]_{\mathrm{i}, \mathrm{j}}=[\mathrm{OR}]_{\mathrm{i}, \mathrm{j}+1} \\
1 & \text { otherwise }
\end{array}\right]
$$

Step 5: First element in [GSM] is set to be equal to the value of the first pixel of [OR]

Step 6: The rest of the elements of [GSM] are calculated as follows:

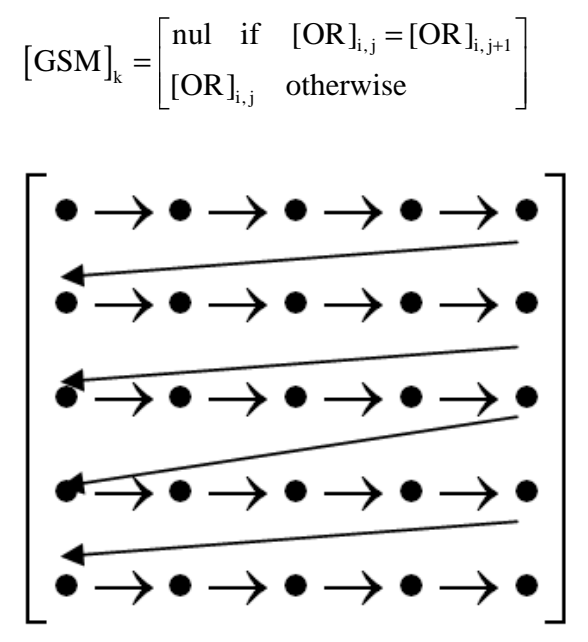

Fig. 1: Original image pixels comparison
Step 7: The original image can be reconstructed as follows:

$$
[\text { rec_img }]_{i, j}=\left[\begin{array}{llc}
{[\mathrm{GSM}]_{\mathrm{k}}} & \text { if } & {[\mathrm{BM}]_{\mathrm{i}, \mathrm{j}}=0} \\
{[\mathrm{GSM}]_{\mathrm{k}+1}} & \text { if } & {[\mathrm{BM}]_{\mathrm{i}, \mathrm{j}}=1}
\end{array}\right]
$$

The performance of the proposed algorithm is compared with other research results and predefined software such as JPEG Lossless Encoder. The criteria used for the comparison is the compression ratio achieved which is defined as follows ${ }^{[4]}$ :

Compression Ratio $(\mathrm{CR})=\frac{\text { Original file size }}{\text { Compressed file size }}$

\section{RESULTS}

The proposed algorithm was implemented using MATLAB $^{[31]}$. The results are obtained from testing on MRI and CT scan images. The original images are grayscale images. The performance of the proposed algorithm is compared with other research results. The results comparisons are shows in Table 3 and 4. The original and reconstructed images are shown in Fig. 2 and 3 respectively.

Table 3: Performance comparison of proposed algorithm and

\begin{tabular}{|c|c|c|}
\hline Method & Technique & Performance \\
\hline${ }_{\text {Xiaofeng } \text { et al., } \text { method }^{[22]}}$ & DPCM and $\mathrm{HC}$ & $1.64-3.12$ \\
\hline $\mathrm{Ng}$ and Cheng method ${ }^{[32]}$ & Sub-block interchange & $1.44-1.52$ \\
\hline Chang and Lin method ${ }^{[33]}$ & Two-way smaller difference & $1.64-1.70$ \\
\hline S.Ganget al., method ${ }^{[34]}$ & DVQ and SPIHT coding & 2.75 \\
\hline Yong et al., method ${ }^{335]}$ & Interval number method & $2.12-2.38$ \\
\hline Lurawave software $^{[36]}$ & Wavelets-LS & $1.49-2.84$ \\
\hline JPEG encoder software ${ }^{[37]}$ & JPEG-LS & $2.71-2.98$ \\
\hline Proposed algorithm & Binary and gray scale matrix & $3.75-4.06$ \\
\hline
\end{tabular}
existing methods in terms of compression ratio

Table 4: Performance comparison of proposed algorithm and existing

\begin{tabular}{|c|c|c|}
\hline Method & Technique & Performance (bpp) \\
\hline Yao method $^{[26]}$ & $\begin{array}{l}\text { Row by row classification } \\
\text { and LZW coding }\end{array}$ & 6.60 \\
\hline Zhang method ${ }^{[38]}$ & Neighborhood block matching & 4.54 \\
\hline $\begin{array}{l}\text { Weinberger et al. }{ }^{[39]} \\
\text { method }\end{array}$ & LOCO-I & 4.04 \\
\hline Proposed algorithm & Binary and gray scale matrix & 2.00 \\
\hline
\end{tabular}
methods in terms of number of bits per pixel

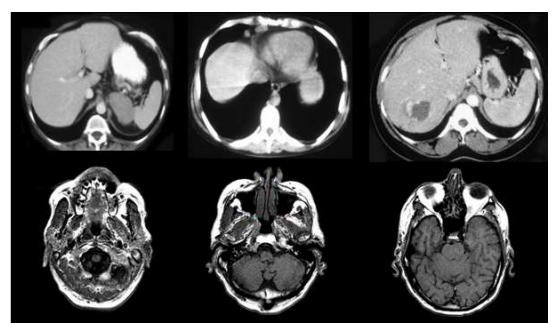

Fig. 2: Original CT and MRI test images 


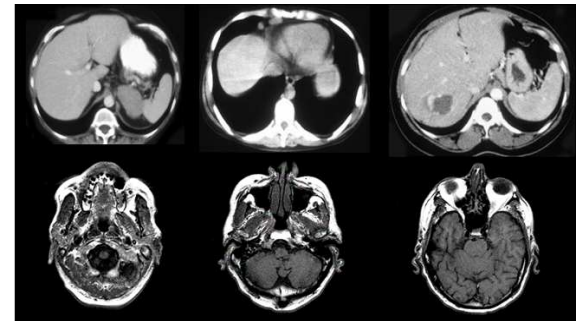

Fig. 3: Reconstructed CT and MRI Images using proposed algorithm

\section{DISCUSSION}

From Table 3 it can be seen that the achieved compression ratio of the proposed algorithm is equal or better than the compression ratios achieved using existing lossless compression techniques. Moreover, Table 4 shows that the number of bits required to represent each pixel is much less compared with the other methods. Finally, Fig. 2 and 3 shows that the quality of the reconstructed images is identical to the original images. The absolute difference of original and reconstructed image has been calculated and the obtained result was all zeros, which means that there is no loss and indicating perfect reconstruction.

\section{CONCLUSION}

In this study an efficient, simple lossless image coding technique is proposed with a remarkable compression ratio and greatly reduced computation load while keeping low complexity compared with other methods. It is very useful for medical images where disease diagnostic requires images to be at as high resolution as possible. It can also be useful for teleradiology and archiving purposes.

\section{REFERENCES}

1. Abdullah, B.J., K.H. Ng and R. Pathmanathen, 1999. The impact of teleradiology in clinical practice-A Malaysian perspective. Med. J. Malaysia, $\quad 54:$ 169-174. http://www.ncbi.nlm.nih.gov/pubmed/10972025

2. Uchida, K., K. Nakamura and H. Watanabe, 1996. Clinical evaluation of irreversible data compression for computed radiography in excretory urography. J. Digit. Imag., 9: 145-149. http://www.ncbi.nlm.nih.gov/pubmed/8854265

3. Streams, S.D., L. Tan and N. Magotra, 1993. Lossless compression of waveform data for efficient storage and transmission. IEEE Trans. Geosci. Remote Sens., 3I: 645-654. http://cat.inist.fr/?aModele=afficheN\&cpsidt=4851127
4. Gonzalez, R. and R. Woods, 2002. Digital Image Processing. 2nd Edn., Prentice Hall, New Jersey, USA., ISBN: 10: 0201180758, pp: 793.

5. Carbal, J.E. and Y. Kim, 1996. Multimedia systems for telemedicine and their communication requirments. IEEE Commun. Mag., 34: 20-27. DOI: $10.1109 / 35.526884$

6. Subhasis Saha, 2000. Image compression-from DCT to wavelets: A review. http://www.acm.org/crossroads/xrds63/sahaimgcoding.html

7. Bauman, R., G. Gell and Dwyer, 1996. Large picture archiving and communication systems of the world- part I. J. Digit. Imag., 9: 99-103. http://www.ncbi.nlm.nih.gov/pubmed/8854258

8. Mark, A., 1998. Image data compression. J. Digit. Imaging, 11: 230-232.

9. Sayood, K., 2000. Introduction to Data Compression. 2nd Edn., Morgan Kaufmann, San Francisco, USA., ISBN: 1558605584, pp: 636.

10. MacMahon, H., K. Doi and S. Sanada, 1991. Data compression: Effect on diagnostic accuracy in digital chest radiography. Radiology, 178: 175-179. http://www.ncbi.nlm.nih.gov/pubmed/1984299

11. Gillespy, T. and A. Rowberg, 1994. Displaying radiological images on personal computers: Image storage and compression. Part 2. J. Digit. Imag., 7: 1-12. http://www.ncbi.nlm.nih.gov/pubmed/8172973

12. Breeuwer, B., R. Heusdens, K. Gunnewiek, P. Zwart and P. Hass, 1995. Data compression of x-ray cardio angiographic image series. Int. J. Card. Imag., 11 : http://www.ncbi.nlm.nih.gov/pubmed/7594747

13. Erickson, B., A. Manduca and P. Person, 1997. Evaluation of irreversible compression of digitized chest radiographs. J. Digit. Imag., 10: 97-102. http://www.ncbi.nlm.nih.gov/pubmed/9268903

14. Zhang, C.N. and X. Wu, 1999. A hybrid approach of wavelet packet and directional decomposition for image compression. Proceeding of IEEE Canadian Conference on Electrical and Computer Engineering, May 9-12, IEEE Xplore Press, USA., pp: 755-780. DOI: 10.1109/CCECE.1999.808039

15. Savcenko, V., B. Erickson and K. Persons, 2000. An evaluation of JPEG and JPEG-2000 irreversible compression algorithms applied to neurologic computed tomography and magnetic resonance images. J. Digit. Imag., 13: 183-185. http://www.ncbi.nlm.nih.gov/pubmed/10847394

16. Saffor, A., A. Ramli and K. Ng, 2001. A comparative study of image compression between JPEG and wavelet. Malaysian J. Comput. Sci., 14: 39-45.

http://mjcs.fsktm.um.edu.my/downlog.asp?AID=112 
17. Saffor, A., A. Ramli and K. Ng, 2002. Objective and subjective evaluation of compressed computed tomography CT images. Internet J. Radiol., 2: 1-5. http://www.ispub.com/journal/the_internet_journal _of_medical_simulation/volume_1_number_1_41/ article/objective_and_subjective_evaluation_of_co mpressed_computed_tomography_ct_images.html

18. Hui, T. and R. Besar, 2002. Medical image compression using JPEG2000 and JPEG: A comparative study. World Sci. J. Mech. Med. Biol., 2: 313-328. http://www.worldscinet.com/cgibin/details.cgi?id=pii:S021951940200054X\&type $=\mathrm{html}$

19. Carlender, M.L. and C. Christopoulos, 2002. Region of interest coding in JPEG 2000. Signal Process. Image Commun. J., 17: 105-111.

20. Kocsis, O., L. Costardidou, L. Varaki, C. Kalogeroulou and G. Panayiotakis, 2003. Compression assessment based on medical image quality concepts using computer generated test images. Comput. Methods Programs Biomed., 71: 105-115. http://www.ncbi.nlm.nih.gov/pubmed/12758132

21. Suryanarayanan, S., A. Karellas, S. Vedentham, S. Waldrop and C. D'Orsi, 2004. A perceptual evaluation of JPEG 2000 image compression for digital mammography: Contrast-detail characteristics. J. Digit. Imag., 17: 64-70. http://www.ncbi.nlm.nih.gov/pubmed/15255520

22. Xiaofeng Li, Yi Shen and Jiachen Ma, 2005. An efficient medical image compression scheme. Proceedings of IEEE Transactions on Engineering in Medicine and Biology 27th Annual Conference, Jan. 17-18, IEEE Xplore Press, Shanghai, China, pp: 3437-3439. DOI: 10.1109/IEMBS.2005.1617217

23. Asraf, R., M. Akbar and N. Jafri, 2006. Statistical analysis of difference image for absolute lossless compression of medical images. Proceedings of the 28th IEEE EMBS Annual International Conference, Aug. 30-Sept. 3, New York City, USA., pp: 4767-4770. DOI: 10.1109/IEMBS.2006.260427

24. Kil, S.K., J.S. Lee, D.F. Shen, J.G. Rye, E.H. Lee, H.K. Min and S.H. Hong, 2006. Lossless medical image compression using redundancy analysis. Int. J. Comput. Sci. Network Secur., 6: 50-56. http://paper.ijcsns.org/07_book/200601/200601A08.pdf

25. Thanoon, B.N., 2007. Using wavelet transform, DPCM and adaptive run-length coding to compress images. Proceedings of the IEEE 6th International Conference on Computer Information Systems and Industrial Management Applications, June 28-30, IEEE Xplore Press, USA., pp: 305-309. DOI: 10.1109/CISIM.2007.74
26. Yao, X., T. Xiao and S. Mao, 2008. Image compression based on classification row by row and LZW encoding. Proceedings of the Congress on Image and Signal Processing, May 27-30, IEEE Xplore Press, China, pp: 617-621. DOI: 10.1109/CISP.2008.302

27. RSNA., 1999. Magnetic resonance imaging. Radiological Society of North America Inc. RSNA. http://www.radiologyinfo.org/content/mr_of_the_b ody.htm

28. RSNA., 2002. Positron emission tomography. Radiological Society of North America Inc. RSNA. http://www.radiologyinfo.org/content/petomograph y.htm

29. Dowsett, D.J., P.A. Kenny and R.E. Johnston, 2006. The Physics of Diagnostic Imaging. 2nd Edn., Hodder Arnold an Imprint of Hodder Education, London, UK., ISBN: 13: 9780340808917 pp: 512.

30. Huang, H.K., 1999. PACS: Basic Principle and Application. John Wiley and Sons, New York, USA.

31. Gonzalez, R., R. Woods and S. Eddins, 2004. Digital Image Processing Using MATLAB. International Edn., Prentice Hall, pp: 624.

32. Ng, K.S. and L.M. Cheng, 1999. Sub-block interchange for lossless image compression. IEEE Trans. Consum. Elect., 45: 236-242. DOI: 10.1109/30.754441

33. Chang, C., C.S. Chan and J. Yuan, 2003. Lossless image compression based on two-way smaller difference. Proceedings of the 17th International Conference on Advanced Information Networking and Application, Mar. 27-29, IEEE Xplore Press, China, pp: 821-824. DOI: 10.1109/AINA.2003.1193008

34. Gang, S., S. Chen and S. Chao, 2003. Waveletbased lossy-to-lossless medical image compression using Dynamic VQ and SPIHT coding. J. Biomed. Eng. Applied Basis Commun., 15: 235-242. http://cat.inist.fr/?aModele $=$ afficheN\&cpsidt $=1540$ 5648

35. Xue Yong, M.M. Rees and X. Sheng, 1998. A simple lossless compression method: Interval number method. Proceeding of the 20th Annual International Conference of the IEEE Engineering in Medicine and Biology Society, Oct. 29-Nov. 1, IEEE Xplore Press, Hong Kong, pp: 1326-1328. DOI: 10.1109/IEMBS.1998.747123

36. Lura Tech 2008. Lura Tech Gmbl. http://www.Luratech.com

37. University of British Colombia JPEG-LS Encoder 1.0. http://www.stat.columbia.edu/ jakulin/jpegls/mirro r.htm 
38. Zhang, L., Y. Quan and B. Zeng, 2005. A lossless compression method of medical images based on neighborhood's match. Proceedings of the International Conference of Machine Learning and Cybernetics, Aug. 18-21, IEEE Xplore Press, Guangzhou, China, pp: 5185-5187. DOI: 10.1109/ICMLC.2005.1527858
39. Weinberger, M.J., G. Seroussi and G. Sapiro, 1996. LOCO-I: A low complexity, context-based, lossless image compression algorithm. Proceedings of Data Compression Conference, Mar.31-Apr. 3, IEEE Xplore Press, Snowbird, UT., USA., pp: 140-149. DOI: 10.1109/DCC.1996.488319 